\title{
Changes in Activation of Abdominal Muscles at Selected Angles During Trunk Exercise by Using Ultrasonography
}

\author{
Hyun-Dong Kim, MD, PhD, Dong-Min Jeon, MD, Hyun-Woo Bae, MD, \\ Jong-Gill Kim, MD, PhD, Nami Han, MD, PhD, Mi-Ja Eom, MD, PhD \\ Department of Physical Medicine and Rehabilitation, Inje University Busan Paik Hospital, \\ Inje University College of Medicine, Busan, Korea
}

Objective To investigate the changes of activation of the abdominal muscles depending on exercise angles and whether the activation of rectus abdominis differs according to the location, during curl up and leg raise exercises, by measuring the thickness ratio of abdominal muscles using ultrasonography.

Methods We examined 30 normal adults without musculoskeletal problems. Muscle thickness was measured in the upper rectus abdominis (URA), lower rectus abdominis (LRA), obliquus externus (EO), obliquus internus (IO), and transversus abdominis (TrA), at pre-determined angles $\left(30^{\circ}, 60^{\circ}, 90^{\circ}\right)$ and additionally at the resting angle $\left(0^{\circ}\right)$. Muscle thickness ratio was calculated by dividing the resting $\left(0^{\circ}\right)$ thickness for each angle, and was used as reflection of muscle activity.

Results The muscle thickness ratio was significantly different depending on the angles in URA and LRA. For curl up-URA p $=0\left(30^{\circ}<60^{\circ}\right), \mathrm{p}=0\left(60^{\circ}>90^{\circ}\right), \mathrm{p}=0.44\left(30^{\circ}<90^{\circ}\right)$ and LRA $\mathrm{p}=0.01\left(30^{\circ}<60^{\circ}\right), \mathrm{p}=0\left(60^{\circ}>90\right)^{\circ}, \mathrm{p}=0.44\left(30^{\circ}>90^{\circ}\right)$, respectively, by one-way ANOVA test-and for leg raise-URA p $=0\left(30^{\circ}<60^{\circ}\right), p=0\left(60^{\circ}<90^{\circ}\right), p=0\left(30^{\circ}<90^{\circ}\right)$ and LRA $\mathrm{p}=0.01\left(30^{\circ}<60^{\circ}\right), \mathrm{p}=0\left(60^{\circ}<90^{\circ}\right), \mathrm{p}=0\left(30^{\circ}<90^{\circ}\right)$, respectively, by one-way ANOVA test-exercises, but not in the lateral abdominal muscles (EO, IO, and TrA). Also, there was no significant difference in the muscle thickness ratio of URA and LRA during both exercises. In the aspect of muscle activity, there was significant difference in the activation of RA muscle by selected angles, but not according to location during both exercises.

Conclusion According to this study, exercise angle is thought to be an important contributing factor for strengthening of RA muscle; however, both the exercises are thought to have no property of strengthening RA muscle selectively based on the location.

Keywords Ultrasonography, Abdominal muscles, Exercise

Received April 6, 2015; Accepted July 6, 2015

Corresponding author: Dong-Min Jeon

Department of Physical Medicine and Rehabilitation, Inje University Busan Paik Hospital, Inje University College of Medicine, 75 Bokji-ro, Busanjingu, Busan 47392, Korea

Tel: +82-51-890-6754, Fax: +82-51-891-1430, E-mail: claudjun@naver.com

(c) This is an open-access article distributed under the terms of the Creative Commons Attribution Non-Commercial License (http://creativecommons.org/ licenses/by-nc/4.0) which permits unrestricted noncommercial use, distribution, and reproduction in any medium, provided the original work is properly cited. Copyright $\odot 2015$ by Korean Academy of Rehabilitation Medicine 


\section{INTRODUCTION}

Abdominal muscle is one of the most important muscles in rehabilitation of patients with musculoskeletal or neurological problems, and has a variety of functions including body movement, posture control, balance, back pain control and supports for coordination of upper and lower limb movements [1]. Thus, various exercises are used in different clinical situations in order to strengthen the abdominal muscles. Of those exercises, curl up or leg raise exercise is one of the commonly performed trunk exercises which are publicly well known, and the procedures are not difficult [2-4]. However, there are no standard sets for the exercise angles formed by the floor and trunk during the curl up exercise, or by the floor and leg during leg raise exercises. Therefore, the exercise angles differ and are dependent on the performers, or at each trial. If the activation of the abdominal muscles varies with the angle during the two exercises, it can be concluded that the exercise angle is a contributing factor for strengthening of the abdominal muscles. Most of the previous studies have been focused on what kind of exercise is more helpful in strengthening the particular abdominal muscle, by comparing the muscle activities between the kinds of abdominal exercises $[2,5,6]$. It is important to choose an exercise type for strengthening the abdominal muscles; however, it is thought that it would be helpful in strengthening the abdominal muscles and clinically necessary to understand and take into account the factors affecting muscle activities when prescribing the same kind of exercise. Recently, some papers have articles based on these topics $[7,8]$. Thus, in this study, we compared the levels of activation of the abdominal muscles depending on angles during the two exercises, by measuring the thickness ratio of abdominal muscles using ultrasonography (USG).

There are several studies that divided the rectus abdominis (RA) muscle into an upper part and a lower part, before comparing the levels of muscle activation accord- ing to location during the two exercises, using electromyography (EMG) [3,4,9-11]. However, the results of previous studies were not coincident and we could not find a study using USG. It is considered to be clinically helpful in prescribing individualized exercises for persons relatively weak in particular parts of RA muscle to understand whether the RA muscle is selectively activated according to location; therefore, we thought that a study using USG was necessary. We also compared the activation of upper RA with lower RA during the two exercises using USG, after which we compared our results and the results of previous studies using EMG.

This study aimed to identify whether the exercise angle could be an important contributing factor for strengthening abdominal muscles, and whether the activation of RA is different based on the location, during the two exercises.

\section{MATERIALS AND METHODS}

\section{Participants}

Thirty healthy staff of Busan Paik Hospital were enrolled as subjects in this study, which included 24 men and 6 women. USG was performed on both sides of the abdomen in each subject. Subjects were aged from 27 to 36 years, with an average age of $30.26 \pm 1.99$ years (male, $30.33 \pm 2.09$ years; female, $30.00 \pm 1.67$ years) (Table 1 ). The subjects enrolled were those who could maintain a certain posture while lifting their bodies or legs for over 30 seconds during the two exercises. We excluded subjects with current back pain and history of spinal disorders, including spinal stenosis, disk disease, or spine fracture. We recruited 30 subjects, and none were excluded during the course of this study. The present research was approved by the Institutional Review Board of Inje University Busan Paik Hospital (No. 14-0159).

\section{Study design and setting}

The examination was carried out after the evaluator

Table 1. Physical characteristics of subjects

\begin{tabular}{lcccc}
\hline Sex & Number & Age (yr) & Height (cm) & Weight (kg) \\
\hline Male & 24 & $30.33 \pm 2.09$ & $171.21 \pm 3.35$ & $68.41 \pm 2.55$ \\
Female & 6 & $30.00 \pm 1.67$ & $160.54 \pm 2.67$ & $60.23 \pm 2.12$ \\
Total & 30 & $30.26 \pm 1.99$ & $164.53 \pm 3.54$ & $63.36 \pm 3.15$ \\
\hline
\end{tabular}

Values are presented as mean \pm standard deviation. 
provided the subjects a complete explanation for both the abdominal exercises and the USG procedure, and obtained their informed consent. A physician who had more than two years of professional practice in musculoskeletal ultrasound at the clinic was appointed as an evaluator. LOGIQ E ultrasound (GE Healthcare, Milwaukee, WI, USA) with linear probe (5-12 MHz) was used for USG. The ultrasound probe was placed in a transverse plane along the abdominal wall for RA muscle. On the basis of a point $3 \mathrm{~cm}$ lateral to the umbilicus, the probe was placed at a point $3 \mathrm{~cm}$ vertically up for the upper rectus abdominis (URA) muscle and at a point $3 \mathrm{~cm}$ vertically down for the lower rectus abdominis (LRA) muscle. For lateral abdominal muscles, it was placed in a transverse plane through the center, between inferior angle of rib cage and iliac crest, and also placed at a point $2.5 \mathrm{~cm}$ in front of the mid-axillary line $[12,13]$. The location of the probe was marked by an ink pen, and it was measured at the same location for all measurements. Subjects were asked to lie in the supine position on the examining table. Curl up exercise was done in a hook lying position with $90^{\circ}$ knee flexion angle. Both hands were placed behind the head and fingers were laced together. The subjects were instructed to curl up their upper bodies, lifting their heads and shoulders off the floor, with both the feet being anchored by one practitioner. Leg raise exercise was performed in the supine position, and the subjects were asked to lift both their legs forward, keeping their knees straight as much as possible. The angle was measured by a goniometer, and the center point of it was placed on the greater trochanter of the femur. The angle between a mid-axillary line and a line parallel to the bottom was measured during curl up exercise. The angle along the axis of femur and a line parallel to the bottom was measured during leg raise exercise. The muscle thickness was measured at an angle and at a point using USG for each exercise. The subjects were asked to maintain a certain posture at a selected exercise angle, and the muscle thickness was measured on three parts of unilateral side, including URA, LRA, and lateral abdominal muscles, in 30 seconds. After that, the subjects were allowed to have a sufficient rest of 2 or 3 minutes in the resting position $\left(0^{\circ}\right)$ before the next measurement (Fig. 1).

\section{Measurements}

The thickness of URA, LRA, obliquus externus (EO), obliquus internus (IO), and transversus abdominis ( $\mathrm{TrA}$ ) muscles was measured bilaterally at angles of $0^{\circ}$ (resting), $30^{\circ}, 60^{\circ}$, and $90^{\circ}$. The stopped images at the selected scanning point were obtained by pressing the 'pause' button on the machine. "The cursor was placed on the boundary of fascia which appears to be the hypoechoic layer and separates the muscle into the surface layer and the deep layer on screen and its distance was measured" [14] (Fig. 2). The thickness of the RA muscle was measured in the middle region of its cross-sectional area (the thickest section of this muscle), and this part was usually around the middle of the image. Also, the thickness of the lateral abdominal muscles was measured at the middle of the image [13-15]. The thickness of muscle tended to be measured a little bit differently depending on the respiratory phase; thus, it was determined to be measured at the end of inhalation. Muscle thickness ratio was calculated by dividing the resting $\left(0^{\circ}\right)$ thickness by the thickness measured at angles of $30^{\circ}, 60^{\circ}$, and $90^{\circ}$.
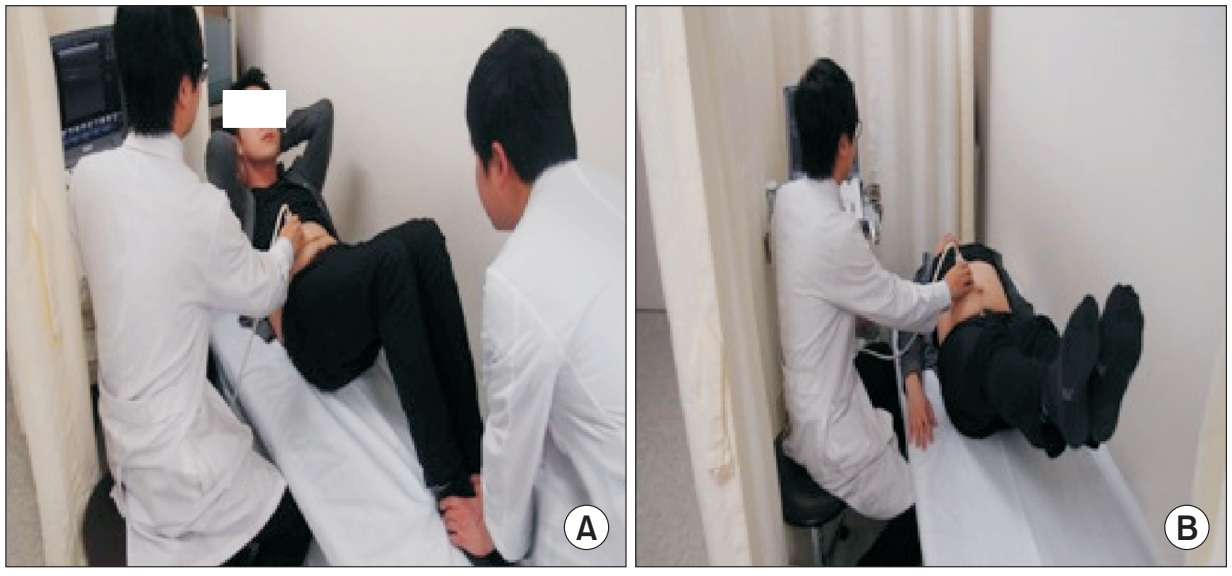

Fig. 1. Images of performing curl up (A) and leg raise (B) exercises while measuring abdominal muscle thickness using ultrasonography. 


\section{Statistical analysis}

The windows SPSS ver. 18.0 (SPSS Inc., Chicago, IL, USA) was used for statistical analysis and the null hypothesis was rejected if the p-value was less than 0.05 . One-way ANOVA test was used to compare the thickness ratio of abdominal muscles at each angle, and the Tukey procedure was used for post hoc tests. In addition, two sample t-tests were used to compare the thickness ratio of URA and LRA.

\section{RESULTS}

The thickness ratio of the abdominal muscles at each angle during curl up exercise is shown in Table 2, and during leg raise exercise in Table 3 . The muscle thickness ratio was significantly different depending on the angles selected for this study $\left(30^{\circ}, 60^{\circ}, 90^{\circ}\right)$ in URA and LRA for curl up exercise and leg raise exercise, but not in the lateral abdominal muscles for curl up exercise and leg raise exercise. In URA and LRA, the highest muscle thickness ratio was measured at $60^{\circ}$ during curl up exercise, and at $90^{\circ}$ during leg raise exercise.

Also, there was no significant difference in the muscle thickness ratio of URA and LRA for curl up exercise$\mathrm{p}=0.35\left(30^{\circ}\right), \mathrm{p}=0.25\left(60^{\circ}\right)$, and $\mathrm{p}=0.13\left(90^{\circ}\right)$ by the twosample t-test - and leg raise exercise $-\mathrm{p}=0.92\left(30^{\circ}\right)$, $\mathrm{p}=0.35\left(60^{\circ}\right)$, and $\mathrm{p}=0.37\left(90^{\circ}\right)$ by the two-sample t-test (Tables 2, 3).

In the aspect of muscle activity, significant difference was seen in the activation of RA muscle by selected angles, but not according to location during both exercises.

\section{DISCUSSION}

Muscle thickness change, as a surrogate marker for the level of muscle activation, was measured using ultrasound in abdominal muscles of 30 healthy adults during curl up and leg raise exercises performed at three dif-
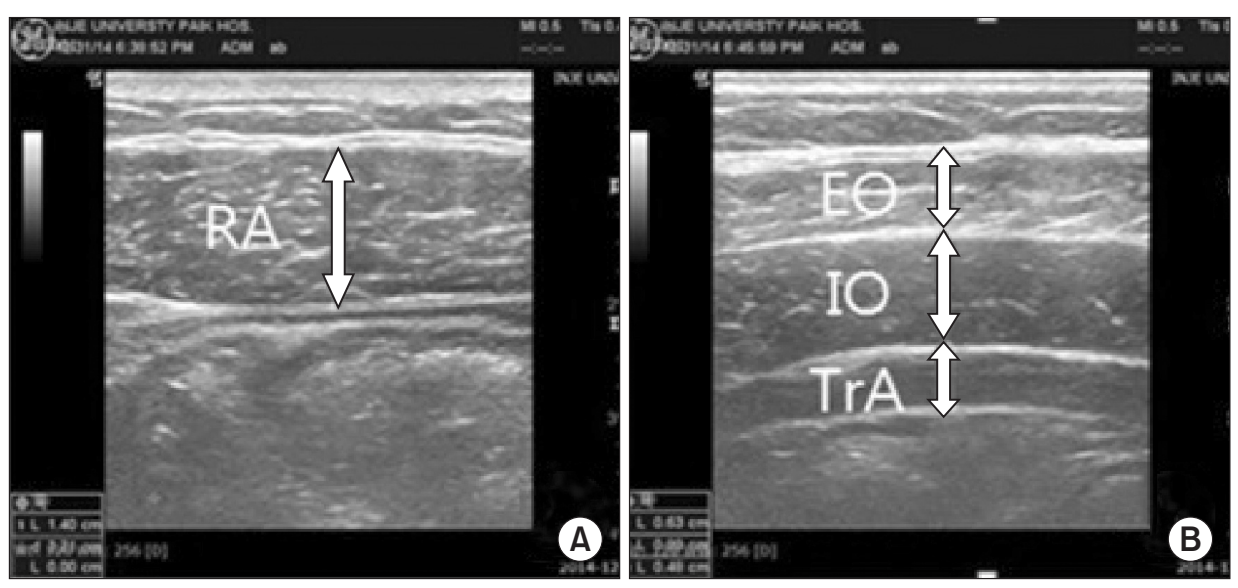

Fig. 2. Ultrasonographic images of rectus (A) and lateral abdominal (B) muscles were made, and thickness was measured from the superficial to deep boundaries of the muscles at the edge of the hypoechoic region. RA, rectus abdominis; EO, obliquus externus; IO, obliquus internus; TrA, transversus abdominis.

Table 2. Curl up exercise: comparison of the thickness ratio of abdominal muscles by the selected angles and location

\begin{tabular}{lccccc}
\hline & $\mathbf{0}^{\circ}$ & $\mathbf{3 0}^{\circ}$ & $\mathbf{6 0}^{\circ}$ & $\mathbf{9 0}^{\circ}$ & p-value $^{\mathrm{a})}$ \\
\hline Upper RA & 1 & $1.38 \pm 0.22$ & $1.60 \pm 0.24$ & $1.43 \pm 0.23$ & $0^{*}\left(0<30^{\circ}, 60^{\circ}, 90^{\circ}\right), 0^{*}\left(30^{\circ}<60^{\circ}\right), 0^{*}\left(60^{\circ}>90^{\circ}\right), 0.44\left(30^{\circ}<90^{\circ}\right)$ \\
Lower RA & 1 & $1.42 \pm 0.22$ & $1.56 \pm 0.19$ & $1.37 \pm 0.19$ & $0^{*}\left(0<30^{\circ}, 60^{\circ}, 90^{\circ}\right), 0.01^{*}\left(30^{\circ}<60^{\circ}\right), 0^{*}\left(60^{\circ}>90^{\circ}\right), 0.44\left(30^{\circ}>90^{\circ}\right)$ \\
p-value $\left.{ }^{b}\right)$ & & 0.35 & 0.25 & 0.13 & \\
EO & 1 & $1.43 \pm 0.30$ & $1.53 \pm 0.38$ & $1.52 \pm 0.33$ & $0^{*}\left(0<30^{\circ}, 60^{\circ}, 90^{\circ}\right), 0.23\left(30^{\circ}<60^{\circ}\right), 0.96\left(60^{\circ}>90^{\circ}\right), 0.35\left(30^{\circ}<90^{\circ}\right)$ \\
IO & 1 & $1.31 \pm 0.26$ & $1.39 \pm 0.33$ & $1.40 \pm 0.34$ & $0^{*}\left(0<30^{\circ}, 60^{\circ}, 90^{\circ}\right), 0.31\left(30^{\circ}<60^{\circ}\right), 0.98\left(60^{\circ}<90^{\circ}\right), 0.23\left(30^{\circ}<90^{\circ}\right)$ \\
TrA & 1 & $1.39 \pm 0.37$ & $1.50 \pm 0.45$ & $1.48 \pm 0.54$ & $0^{*}\left(0<30^{\circ}, 60^{\circ}, 90^{\circ}\right), 0.39\left(30^{\circ}<60^{\circ}\right), 0.97\left(60^{\circ}>90^{\circ}\right), 0.52\left(30^{\circ}<90^{\circ}\right)$ \\
\hline
\end{tabular}

Values are muscle thickness ratio which were calculated by dividing resting $\left(0^{\circ}\right)$ thickness for each angle and presented as mean \pm standard deviation.

RA, rectus abdominis; EO, obliquus externus; IO, obliquus internus; TrA, transversus abdominis.

${ }^{a)} \mathrm{p}$-value was calculated by one-way ANOVA test which compared values according to angles $\left({ }^{*} \mathrm{p}<0.05\right)$.

b) $\mathrm{p}$-value was calculated by two sample t-test which compared values according to location of RA muscle $\left({ }^{*} \mathrm{p}<0.05\right)$. 
Table 3. Leg raise exercise: comparison of the thickness ratio of abdominal muscles by the selected angles and location

\begin{tabular}{lccccc}
\hline & $\mathbf{0}^{\mathbf{o}}$ & $\mathbf{3 0}^{\circ}$ & $\mathbf{6 0}^{\circ}$ & $\mathbf{9 0}^{\circ}$ & p-value \\
\hline Upper RA & 1 & $1.17 \pm 0.13$ & $1.29 \pm 0.17$ & $1.40 \pm 0.12$ & $0^{*}\left(0<30^{\circ}, 60^{\circ}, 90^{\circ}\right), 0^{*}\left(30^{\circ}<60^{\circ}\right), 0^{*}\left(60^{\circ}<90^{\circ}\right), 0^{*}\left(30^{\circ}<90^{\circ}\right)$ \\
Lower RA & 1 & $1.17 \pm 0.11$ & $1.26 \pm 0.16$ & $1.38 \pm 0.11$ & $0^{*}\left(0<30^{\circ}, 60^{\circ}, 90^{\circ}\right), 0.01^{*}\left(30^{\circ}<60^{\circ}\right), 0^{*}\left(60^{\circ}<90^{\circ}\right), 0^{*}\left(30^{\circ}<90^{\circ}\right)$ \\
p-value ${ }^{b)}$ & & 0.92 & 0.35 & 0.37 & \\
EO & 1 & $1.30 \pm 0.27$ & $1.38 \pm 0.28$ & $1.41 \pm 0.20$ & $0^{*}\left(0<30^{\circ}, 60^{\circ}, 90^{\circ}\right), 0.23\left(30^{\circ}<60^{\circ}\right), 0.75\left(60^{\circ}<90^{\circ}\right), 0.06\left(30^{\circ}<90^{\circ}\right)$ \\
IO & 1 & $1.22 \pm 0.23$ & $1.24 \pm 0.20$ & $1.27 \pm 0.23$ & $0^{*}\left(0<30^{\circ}, 60^{\circ}, 90^{\circ}\right), 0.81\left(30^{\circ}<60^{\circ}\right), 0.72\left(60^{\circ}<90^{\circ}\right), 0.35\left(30^{\circ}<90^{\circ}\right)$ \\
TrA & 1 & $1.38 \pm 0.49$ & $1.48 \pm 0.56$ & $1.43 \pm 0.53$ & $0^{*}\left(0<30^{\circ}, 60^{\circ}, 90^{\circ}\right), 0.53\left(30^{\circ}<60^{\circ}\right), 0.84\left(60^{\circ}>90^{\circ}\right), 0.86\left(30^{\circ}<90^{\circ}\right)$ \\
\hline
\end{tabular}

Values are muscle thickness ratio which were calculated by dividing resting $\left(0^{\circ}\right)$ thickness for each angle and presented as mean \pm standard deviation.

RA, rectus abdominis; EO, obliquus externus; IO, obliquus internus; TrA, transversus abdominis.

${ }^{a)} \mathrm{p}$-value was calculated by one-way ANOVA test which compared values according to angles $\left({ }^{*} \mathrm{p}<0.05\right)$.

${ }^{b)} \mathrm{p}$-value was calculated by two sample t-test which compared values according to location of RA muscle $\left({ }^{*} \mathrm{p}<0.05\right)$.

ferent angles. Depending on different exercise angles, significant changes of muscle thickness was shown in the URA and LRA, but not in the lateral abdominal muscles. The activation levels of URA and LRA were not substantially affected by the two different abdominal exercises.

Based on the results of this study, the exercise angle is thought to be an important contributing factor for strengthening of RA muscle, because the muscle thickness ratio was significantly different, depending on the angles selected for this study $\left(30^{\circ}, 60^{\circ}, 90^{\circ}\right)$ in RA muscle. The highest muscle thickness ratio was measured at $60^{\circ}$ during curl up exercise, and at $90^{\circ}$ during leg raise exercise. It is clear that this study has some limitations in that, while not utilizing EMG, we measured the muscle activities by USG alone, and compared between the selected particular angles; however, it is thought that at least, increasing the exercise angle to more than $30^{\circ}$ when doing the two exercises would be helpful in strengthening the RA muscle.

For the lateral abdominal muscles, no significant difference was found in muscle thickness ratio depending on the selected angles $\left(30^{\circ}, 60^{\circ}, 90^{\circ}\right)$ during the two exercises. We considered that lateral abdominal muscles might mainly act on the maintenance of trunk stability instead of the exercise motion itself, leading to the results differing from those of the RA muscle $[6,12,16]$. In addition, we thought that there might be differences in muscle activities depending on the angles when doing the exercises, including trunk rotation or lateral bending motions; it is considered that additional studies are needed to be conducted in the future.

Some studies have been reported recently on the fac- tors affecting the abdominal muscle activation when performing the curl up exercise [7,8]. In this study, we only examined whether the exercise angle is a contributing factor; however, we thought that exercise velocity or the presence of resistance at the time of exercise could also be factors that affect strengthening the abdominal muscles. It is expected that more helpful exercise prescriptions will be possible when prescribing similar exercises, if the studies on the various factors that affect strengthening the abdominal muscles are to be conducted continuously in the future.

We did not observe any difference in the muscle thickness ratio of URA and LRA at each angle in both exercises in this study. According to previous studies, the RA muscle is dominated by not only common nerve branch but also other nerve branches. In other words, each part of RA is controlled by a common part and at the same time, influenced by an independent part [3]. Based on the above findings, there were several papers which compared the levels of muscle activation using EMG, under the assumption that there might be difference in the levels of RA muscle activation between upper and lower parts while performing curl up or leg raise up exercises [3,4,9-11]. Several studies showed that the URA was more activated than the LRA during curl up exercise; however, the LRA was more activated during leg raise exercise. On the other hand, another study reported that there was no difference in the levels of muscle activation between the upper and lower parts of RA muscle during the two exercise regimens. Subjects, measurement locations, procedures and measurement devices are different in each study. Therefore, a large-scale study in future, which 
considers this risk of bias, is thought to be required. If difference in activation of upper and lower RA during trunk exercises is scientifically proven in future studies, individualized exercise can be prescribed for strengthening the relatively weak portion of RA muscle intensively.

In this study, we used USG to compare muscle activity instead of EMG. In many previous studies, abdominal muscle activation was evaluated by measuring the signal amplitude of EMG with surface EMG electrodes or needle electrodes. However, USG can also be a useful tool for evaluation, and has some advantages compared to EMG. Very few studies reported that it can be evaluation can be done based on changes in the thickness of abdominal muscles measured by USG. One study showed that changes in the thickness of TrA and IO muscles, as seen on ultrasound imaging, have statistically significant relation to the recruitment of TrA and IO muscles, as seen on EMG [14]. Another study reported that the relationship between TrA muscle activation measured by EMG and its thickness change measured by USG was statistically significant [15]. According to the study by Hodges et al. [17], they ascertained that USG quantifies morphological changes in the muscle, including a change in thickness, and this quantified value can be a mean to define the levels of muscle activation. One more study showed that the relative change of the muscle thickness could be used as the indicator of the muscle activity in $\operatorname{TrA}$ and lumbar multifidus muscles [18]. Two other studies also used USG as a tool for measuring abdominal muscle activation $[6,12]$. Moreover, USG has several advantages compared to EMG. First, it is a non-invasive procedure and easy to perform when compared to needle EMG. Second, USG is able to measure changes in the abdominal muscle in a large area, and not just in a localized area. EMG is capable of detecting only those signals close to surface EMG electrodes or needle electrodes in a localized area. Third, needle EMG has a potential risk for subjects, such as pain and infection. Also, some factors exist that can influence the magnitude of the surface EMG signal. These factors include subcutaneous tissue under the electrodes, and muscle movement relative to the electrodes [4]. In addition, USG turned out to be a reliable test in several studies because there was no statistically significant difference in the results of measuring the muscle thickness between the evaluators and during repeated measurements $[6,12,14]$. Although, it is still controversial whether
USG is able to fully reflect the actual levels of muscle activation or not, we used USG in this study since it is noninvasive, easy to perform, and can reflect muscle activation of larger area than EMG.

In this study, standard deviations of the TrA muscle thickness ratio were relatively higher than the results of other muscles during both exercises, but especially during leg raise exercise. That is, the rates of change in muscle thickness were largely different among the subjects. From the results generated, we assumed that there might be differences in the levels of activation of TrA muscle depending on breathing and abdominal movements, as was observed in previous studies. One study reported that the TrA muscle was more highly activated by abdominal draw in maneuver for lower abdominal region, compared to other exercises [5]. In another study, the TrA muscle was highly activated during the peak expiration [6]. Therefore, even if the same exercise is performed, the exercise could be more effective depending on breathing and abdominal movements for TrA muscle.

There are several limitations in this study. First, the sample size of our study was small and most of the subjects were men. Since the subjects were only a small number of young men and women, is the data is insufficient to be generalized. Second, only muscle thickness was measured in this study, instead of other various morphological changes of muscles including crosssectional area, length, and pennation angle. Third, we could not compare the levels of muscle activation appearing on USG and EMG, since we did not use EMG in this study. Further studies are required to measure the levels of muscle activation at different angles during both the above exercises using EMG, and compare with the results of this study. Lastly, we did not measure the muscle thickness by subdividing exercise angles more in detail during both exercises.

Based on the results of this study, the exercise angle is thought to be an important contributing factor for strengthening of RA muscle. Both exercises are thought to have no property of strengthening RA muscle selectively according to location.

\section{CONFLICT OF INTEREST}

No potential conflict of interest relevant to this article was reported. 


\section{REFERENCES}

1. Kibler WB, Press J, Sciascia A. The role of core stability in athletic function. Sports Med 2006;36:189-98.

2. Willett GM, Hyde JE, Uhrlaub MB, Wendel CL, Karst GM. Relative activity of abdominal muscles during commonly prescribed strengthening exercises. J Strength Cond Res 2001;15:480-5.

3. Marchetti PH, Kohn AF, Duarte M. Selective activation of the rectus abdominis muscle during low-intensity and fatiguing tasks. J Sports Sci Med 2011;10:322-7.

4. Lehman GJ, McGill SM. Quantification of the differences in electromyographic activity magnitude between the upper and lower portions of the rectus abdominis muscle during selected trunk exercises. Phys Ther 2001;81:1096-101.

5. Urquhart DM, Hodges PW, Allen TJ, Story IH. Abdominal muscle recruitment during a range of voluntary exercises. Man Ther 2005;10:144-53.

6. Ishida $\mathrm{H}$, Hirose $\mathrm{R}$, Watanabe $\mathrm{S}$. Comparison of changes in the contraction of the lateral abdominal muscles between the abdominal drawing-in maneuver and breathe held at the maximum expiratory level. Man Ther 2012;17:427-31.

7. Kim MH, Oh JS. Effects of performing an abdominal hollowing exercise on trunk muscle activity during curl-up exercise on an unstable surface. J Phys Ther Sci 2015;27:501-3.

8. Yoon TL, Kim KS, Cynn HS. Slow expiration reduces sternocleidomastoid activity and increases transversus abdominis and internal oblique muscle activity during abdominal curl-up. J Electromyogr Kinesiol 2014;24:228-32.

9. Sarti MA, Monfort M, Fuster MA, Villaplana LA. Muscle activity in upper and lower rectus abdominus during abdominal exercises. Arch Phys Med Rehabil
1996;77:1293-7.

10. Duncan M. Muscle activity of the upper and lower rectus abdominis during exercises performed on and off a Swiss ball. J Bodyw Mov Ther 2009;13:364-7.

11. Clark KM, Holt LE, Sinyard J. Electromyographic comparison of the upper and lower rectus abdominis during abdominal exercises. J Strength Cond Res 2003;17:475-83.

12. Critchley DJ, Coutts FJ. Abdominal muscle function in chronic low back pain patients: measurement with real-time ultrasound scanning. Physiotherapy 2002;88:322-32.

13. Rankin G, Stokes M, Newham DJ. Abdominal muscle size and symmetry in normal subjects. Muscle Nerve 2006;34:320-6.

14. Ferreira PH, Ferreira ML, Nascimento DP, Pinto RZ, Franco MR, Hodges PW. Discriminative and reliability analyses of ultrasound measurement of abdominal muscles recruitment. Man Ther 2011;16:463-9.

15. Ferreira PH, Ferreira ML, Hodges PW. Changes in recruitment of the abdominal muscles in people with low back pain: ultrasound measurement of muscle activity. Spine (Phila Pa 1976) 2004;29:2560-6.

16. Stokes IA, Gardner-Morse MG, Henry SM. Abdominal muscle activation increases lumbar spinal stability: analysis of contributions of different muscle groups. Clin Biomech (Bristol, Avon) 2011;26:797-803.

17. Hodges PW, Pengel LH, Herbert RD, Gandevia SC. Measurement of muscle contraction with ultrasound imaging. Muscle Nerve 2003;27:682-92.

18. Djordjevic O, Konstantinovic L, Miljkovic N, Bijelic G. Relationship between electromyographic signal amplitude and thickness change of the trunk muscles in patients with and without low back pain. Clin J Pain 2015;31:893-902. 